\title{
Measurements of thermal fission and capture cross sections of minor actinides within the Mini-INCA project
}

\author{
O. Bringer ${ }^{1}$, I. AlMahamid ${ }^{5}$, S. Chabod ${ }^{1}$, F. Chartier ${ }^{3}$, E. Dupont ${ }^{1}$, A. Letourneau ${ }^{1, a}$, P. Mutti ${ }^{4}$, L. Oriol ${ }^{2}$, \\ S. Panebianco ${ }^{1}$, and Christian Veyssière ${ }^{1}$ \\ ${ }^{1}$ CEA/DSM/DAPNIA-Saclay, 91191 Gif-sur-Yvette, France \\ 2 CEA/DEN/DER-Cadarache, 13108 Saint-Paul-lez-Durance, France \\ 3 CEA/DEN/DPC-Saclay, 91191 Gif-sur-Yvette, France \\ ${ }^{4}$ Institut Laue Langevin, 38000 Grenoble, France \\ 5 Wadsworth Center, New York State Department of Health, Albany, NY 12201, USA
}

\begin{abstract}
In the framework of nuclear waste transmutation studies, the Mini-INCA project has been initiated at CEA/DSM to determine optimal conditions for transmutation and incineration of Minor Actinides (MA) in high intensity neutron fluxes in the thermal region. Our experimental tool is based on alpha- and gamma-spectroscopy of irradiated samples and microscopic fission-chambers. It can provide both microscopic information on nuclear reactions (total and partial cross sections for neutron capture and/or fission reactions) and macroscopic information on transmutation and incineration potentials. The ${ }^{232} \mathrm{Th},{ }^{237} \mathrm{~Np},{ }^{241} \mathrm{Am}$, and ${ }^{244} \mathrm{Cm}$ transmutation chains have been explored in details, showing some discrepancies in comparison with evaluated data libraries but in overall good agreement with recent experimental data.
\end{abstract}

\section{Introduction}

Faced with the growing world power demands, nuclear energy seems to be a conceivable solution for energy production without using remaining limited fossil fuel resources which contribute to the alarming global warming effect. However, public opinion still expresses major concerns about this energy source, mainly due to the residual long-term highly radioactive wastes. In this context, even if the creation of a long-term geological repository seems to be unavoidable, researches and developments about partitioning and transmutation are essentials. In particular, the reduction of uncertainties on nuclear data is one of the fundamental aspects of these researches for systems considered as possible transmuters of transuranic elements or even simply for systems which have to respond to high-level requirements as waste minimization, sustainability, safety and non-proliferation. Most of the nuclear data are available in modern data files, but their accuracies and validation are still a major concern, especially for Minor Actinides (MA).

Indeed, a recent study was performed regarding the impact of nuclear data uncertainties on performance parameters (criticality, reactivity, irradiated fuel isotopic composition, neutron sources...) of future nuclear systems and has been done in some details [1]. This study concludes that MA data uncertainties don't play a major role on essential nuclear parameters such as fuel burn-up or criticality. However, when considering the precision of key transmutation parameters [2] such as the incineration potential and the production of $\alpha$ or neutron-emitters, which has to do with the economy and safety of the installation, inaccuracies could play a much more important role. This is true for high burn-up or multirecycled fuels within GEN III reactors.

\footnotetext{
${ }^{a}$ Presenting author, e-mail: aletourneau@cea.fr
}

Within the Mini-INCA project [3], at the French Atomic Energy Commission (CEA), we have developed experimental tools and techniques to provide reliable data on MA in the thermal energy region. In this paper, we present the experimental approach and the latest experimental results obtained on fission and capture cross sections involved in ${ }^{232} \mathrm{Th},{ }^{237} \mathrm{~Np}$, ${ }^{241} \mathrm{Am}$, and ${ }^{244} \mathrm{Cm}$ transmutation chains.

\section{Experimental approach}

\subsection{Irradiation facilities}

The High Flux Reactor of Laue Langevin Institut (Grenoble, France) is used to perform MA irradiations. It provides very high intensity flux of thermalized neutrons. Therefore, it is possible to use $\mu \mathrm{g}$-mass samples, resulting in negligible local flux perturbation. In addition, the formation of shortlived isotopes can be studied on-line. Two channels in the reactors have been equipped for irradiations (see fig. 1). The V4 channel provides different neutron energy spectra, corresponding to different positions. In this channel, the thermal neutron proportion $\left(\mathrm{E}_{n}<1 \mathrm{eV}\right)$ varies from $100 \%$ to $85 \%$, and intensities spread from $6 \cdot 10^{13} \mathrm{n} . \mathrm{cm}^{-2} \cdot \mathrm{s}^{-1}$ to $1.5 \cdot 10^{15}$ n. $\mathrm{cm}^{-2} \cdot \mathrm{s}^{-1}$. The H9 channel has a neutron flux close to the one available in an intermediate position of V4, with about $2 \%$ of neutrons having energies greater than $1 \mathrm{eV}$. In some cases, we can also use the T4 channel, which provides a pure thermal neutron flux with an intensity of $2 \cdot 10^{13} \mathrm{n} / \mathrm{cm}^{2} / \mathrm{s}$ [4].

In addition to these quasi-thermal neutron fluxes available at ILL, the MEGAPIE target at Paul-Scherrer Institute (Villingen, Switzerland) was also used to provide a moderated spallation neutron flux [5] to measure the transmutation of ${ }^{241} \mathrm{Am}$ and ${ }^{237} \mathrm{~Np}$ in the epithermal region. 


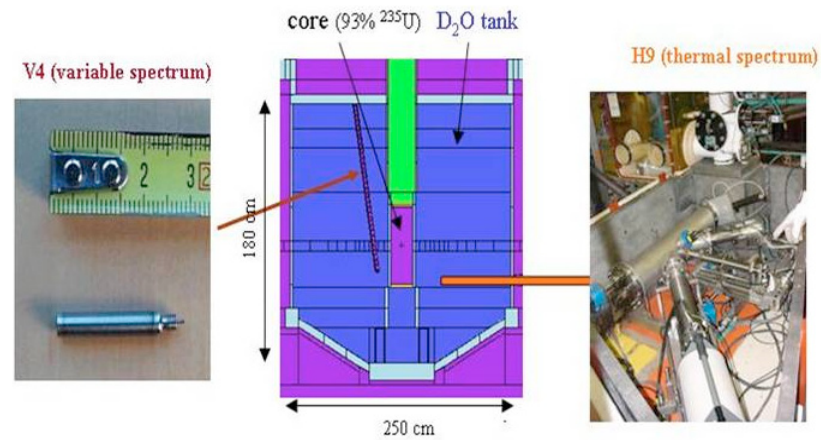

Fig. 1. The Mini-INCA installation at ILL. The reactor core is sketched with two irradiation channels. The experimental tools used in the two channels (a fission chamber for V4 and an alpha- and a gamma-spectroscopy station for $\mathrm{H} 9$ ) are also shown (left and right).

\subsection{Experimental set-up}

As shown in figure 1, two complementary experimental setups have been developed at the ILL reactor. First, a dedicated spectroscopy bench [6] have been installed and connected to the source exchanger of the Lohengrin mass spectrometer (H9 channel). Equipped with $\mathrm{Si}$ - and Ge-detectors, it allows a quasi-on-line characterization of irradiated samples by $\alpha$ and $\gamma$ spectroscopy as well as repeated sequences of irradiation and measurement. The Si- and Ge-detector electronics is able to manage high counting rates (up to $80 \mathrm{kHz}$ for Ge-detector and up to $20 \mathrm{kHz}$ for Si-detector). The $\alpha$ and $\gamma$ spectroscopy bench allows a fast characterization of irradiated samples and a direct access to short-lived isotopes.

Incineration studies in the V4 channel are performed by using dedicated compensated detectors $[7,8]$. They are composed of three microscopic fission-chambers ${ }^{1}$, called TripleDeposit Fission-Chambers (TDFC), mechanically coupled but electrically independent and sharing the same gas. TDFC operates in current mode to measure on-line the actinide fission current with respect to the known ${ }^{235} \mathrm{U}$ fission current. Parasitic current induced by $\gamma$-rays and neutron activation can be subtracted thanks to the third chamber without deposit. Each fission chamber is $2 \mathrm{~cm}$ long, has a diameter of $8 \mathrm{~mm}$, and is filled with pure Argon gas. Therefore, these small detectors could be placed one very close to the other, thus reducing systematic errors due to distance. Space charge effects due to high fission rates (high fluxes) are considerably reduced thanks to the thin gap between anode and cathode. Indeed they can be used in high neutron fluxes up to $8 \cdot 10^{14}$ $\mathrm{n} / \mathrm{cm}^{2} / \mathrm{s}$. Their functioning is detailed in ref [8].

Finally, precise mass spectrometry (Thermal Ionisation and Inductive Coupled Plasma Mass Spectrometry) analysis can be performed after irradiation in V4.

\subsection{Analysis tools}

High fluxes are very useful to explore transmutation chains, i.e., to form and study short-lived isotopes for which targets are impossible to make. On the other hand, the evolution

\footnotetext{
${ }^{1}$ Fabricated by Photonis company.
}

of MA has a very complex scheme and experimental results can be difficult to interpret, especially for long-time irradiations. In this context, we have developed an evolution code called MERCS [9] and used it both for analysis (fit of nuclear parameters on experimental data) and to optimise irradiation conditions (suitable neutron flux and irradiation time). MERCS is a one-group evolution code, based on ROOT shared libraries, which solves numerically Bateman equations. Its originality lies within its capacity to compute absolute and relative sensitivity tables, which reflect the sensitivity of an experimental observable (i.e. fission chamber current) to nuclear parameters.

\section{Integral measurements}

\subsection{Neutron flux}

The MCNP code was used to model the ILL reactor core and to calculate neutron flux distributions at different irradiation positions. Results are shown in figure 2. As the moderator temperature is $(50 \pm 0.5){ }^{\circ} \mathrm{C}$, two calculations were performed: one with libraries at $300 \mathrm{~K}$ and one with libraries at $400 \mathrm{~K}$. The difference in the neutron energy spectra is simply a shift in the Maxwellian distribution to higher neutron energies, corresponding to a temperature of $400 \mathrm{~K}$.

\subsection{Effective and $25.3 \mathrm{meV}$ cross sections}

The data we measure are, in fact, effective cross sections:

$$
\langle\sigma\rangle=\frac{\int_{0}^{\infty} \sigma(E) \cdot \phi(E) d E}{\int_{0}^{\infty} \phi(E) d E}
$$

where $\sigma(\mathrm{E})$ is the cross section as a function of neutron energy and $\Phi(\mathrm{E})$ the neutron energy distribution in the irradiation.

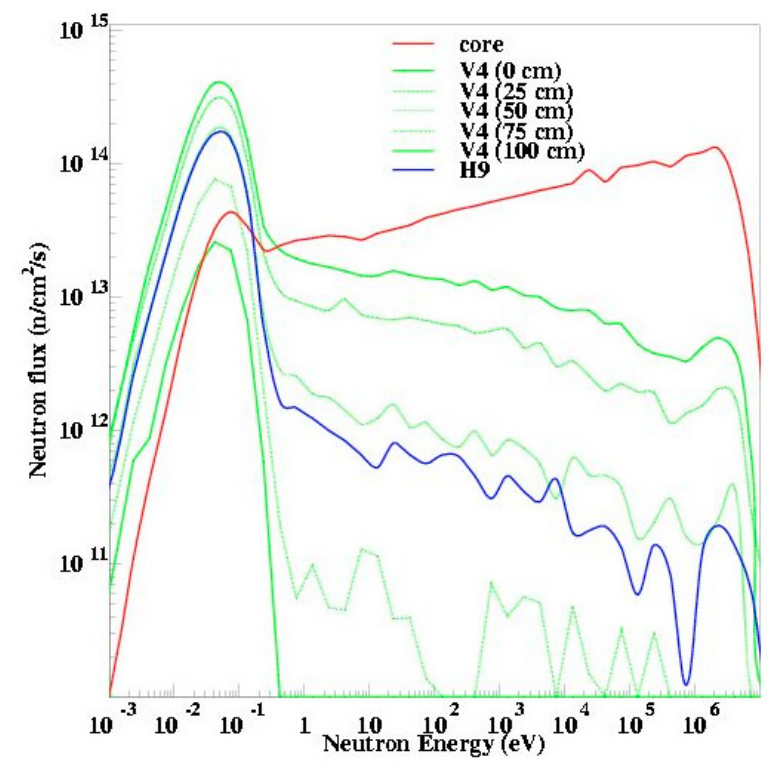

Fig. 2. Neutron energy spectra simulated with MCNP at different positions of irradiation at the HFR [10]. 
Table 1. Effective cross sections of the two normalisation reactions and thermal-over-total neutron flux ratios in different irradiation positions.

\begin{tabular}{llll}
\hline Positions & ${ }^{59} \operatorname{Co}(\mathrm{n}, \gamma){ }^{60} \mathrm{Co}$ & ${ }^{235} \mathrm{U}(\mathrm{n}, \mathrm{f})$ & $\mathrm{R}_{\mathrm{th}} / \mathrm{R}_{\text {tot }}$ \\
\hline $25 \mathrm{~cm}$ & 28.33 & 424.68 & 0.898 \\
$50 \mathrm{~cm}$ & 30.74 & 466.83 & 0.966 \\
$75 \mathrm{~cm}$ & 31.66 & 484.17 & 0.997 \\
$100 \mathrm{~cm}$ & 31.84 & 487.29 & 1.000 \\
$\mathrm{H} 9$ & 31.10 & 473.93 & 0.981 \\
\hline
\end{tabular}

With $\sigma(\mathrm{E})$ from nuclear data libraries and $\Phi(\mathrm{E})$ calculated as previously described, it is possible to extract the effective cross section [11]. As an example, effective cross sections of standard reactions used to normalise the neutron flux are indicated in table 1 . Because $\sigma(\mathrm{E})$ is well described and resonances do not contribute too much to the effective cross section, it is also possible to extract the $25.3 \mathrm{meV}$ value $\left(\sigma_{0}\right)$ with a good precision by using the ratio $\sigma_{0} /\langle\sigma\rangle$ calculated from data bases. When $\sigma(\mathrm{E})$ is not well known, as in the case of shortlived isotopes, this value should be taken as less accurate. Nevertheless, the uncertainties on resonance parameters and on neutron flux distribution are fully propagated through the extraction procedure.

\section{Recent results}

\subsection{Thorium transmutation chain}

${ }^{232} \mathrm{Th}(\mathrm{n}, \gamma){ }^{233} \mathrm{Th}$ and ${ }^{233} \mathrm{~Pa}(\mathrm{n}, \gamma){ }^{234} \mathrm{~Pa}$ reaction cross sections were measured by means of a precise mass spectrometry analysis (Thermal Ionisation and Induced Coupled Plasma Mass spectrometry) [12]. The analysis was performed few months after a 43 day irradiation in V4. The irradiated sample was a pure $100 \mu \mathrm{g}{ }^{232} \mathrm{Th}$ sample canned in a quartz container. The irradiation position was chosen to get a significant evolution by multiple captures (i.e., intense flux) and to reduce corrections due to the contribution of resonances (i.e. thermal flux). The neutron fluency, over which thorium evolved, was measured thanks to an $\mathrm{Al}-0.1 \%{ }^{59} \mathrm{Co}$ monitor.

Experimental uranium and thorium isotopic ratios were fitted using the MERCS code with the effective cross section values listed in table 2 as free parameters. The fit procedure was repeated several times. At each step, all other nuclear parameters (included neutron flux for which we have used the experimental value) were randomly sampled within their Gaussian error distribution. Moreover, every isotopic ratio was chosen to be mainly sensitive to one cross section. By this means, the covariance matrix is closed to a diagonal one and calculated errors could be considered independent. Resulting cross sections are shown in table 2.

Table 2. Effective and $25.3 \mathrm{meV}$ measured cross sections.

\begin{tabular}{lll}
\hline Reactions & $\langle\sigma\rangle(\mathrm{b})$ & $\sigma_{0}(\mathrm{~b})$ \\
\hline$\left.{ }^{232} \mathrm{Th}(\mathrm{n}, \gamma)\right)^{233} \mathrm{Th}$ & $6.27 \pm 0.18$ & $7.3 \pm 0.2$ \\
${ }^{233} \mathrm{~Pa}(\mathrm{n}, \gamma)^{234} \mathrm{~Pa}$ & $34.16 \pm 1.54$ & $38.3 \pm 1.8$ \\
${ }^{234} \mathrm{U}(\mathrm{n}, \gamma)^{235} \mathrm{U}$ & $87.34 \pm 2.75$ & $106 \pm 3$ \\
${ }^{235} \mathrm{U}(\mathrm{n}, \gamma){ }^{236} \mathrm{U}$ & $79.01 \pm 9.05$ & $98 \pm 11$ \\
\hline
\end{tabular}

\subsection{Neptunium transmutation chain}

${ }^{237} \mathrm{~Np}(\mathrm{n}, \gamma){ }^{238} \mathrm{~Np}$ cross section was measured by $\alpha$ and $\gamma$ spectroscopy on the $\mathrm{H} 9$ channel. $13.82 \mu \mathrm{g}$ of ${ }^{237} \mathrm{~Np}$ were electrodeposited on a Ti backing and irradiated for 2 hours in the $\mathrm{H} 9$ channel. In addition, the ${ }^{238} \mathrm{~Np}(\mathrm{n}, \mathrm{f})$ and ${ }^{238} \mathrm{Pu}(\mathrm{n}, \gamma){ }^{239} \mathrm{Pu}$ cross sections were measured in the $\mathrm{V} 4$ channel using a TDFC with a ${ }^{237} \mathrm{~Np}$ deposit of $(42 \pm 1.3) \mu \mathrm{g}$ [13]. TDFC were irradiated for 43 days. The nuclear parameters were fitted for the experimental current using the MERCS code. The resulting cross sections are shown in table 3. Our measured ${ }^{237} \mathrm{~Np}(\mathrm{n}, \gamma){ }^{238} \mathrm{~Np}$ cross section is $10 \%$ larger than ENDF/BVII, JENDL-3.3 and JEFF-3.1, whereas it is compatible with older versions (ENDF/B-VI, JEF-2). Our measured ${ }^{238} \mathrm{~Np}(\mathrm{n}, \mathrm{f})$ is in a good agreement with libraries, whereas the latter overestimates the ${ }^{238} \mathrm{Pu}(\mathrm{n}, \gamma){ }^{239} \mathrm{Pu}$ cross section by $15 \%$. The latter value will be measured again this year.

Table 3. Effective and $25.3 \mathrm{meV}$ measured cross sections.

\begin{tabular}{lll}
\hline Reactions & $\langle\sigma\rangle(\mathrm{b})$ & $\sigma_{0}(\mathrm{~b})$ \\
\hline${ }^{237} \mathrm{~Np}(\mathrm{n}, \gamma){ }^{238} \mathrm{~Np}$ & $150 \pm 4.2$ & $180 \pm 5$ \\
${ }^{238} \mathrm{~Np}(\mathrm{n}, \mathrm{f})$ & $1828 \pm 55$ & $2165 \pm 70$ \\
${ }^{238} \mathrm{Pu}(\mathrm{n}, \gamma){ }^{239} \mathrm{Pu}$ & $388 \pm 12$ & $476 \pm 33$ \\
\hline
\end{tabular}

\subsection{Americium transmutation chain}

The americium transmutation chain was studied in detail using post-irradiation mass-spectrometry analysis for $\quad{ }^{241} \mathrm{Am}(\mathrm{n}, \gamma)^{242 \mathrm{~m}-\mathrm{gs}} \mathrm{Am}, \quad{ }^{242 \mathrm{~m}} \mathrm{Am}(\mathrm{n}, \gamma)^{243} \mathrm{Am} \quad$ and ${ }^{242} \mathrm{Cm}(\mathrm{n}, \gamma){ }^{243} \mathrm{Cm}$ cross sections, and using fission chambers measurement for ${ }^{242 \mathrm{gs}} \mathrm{Am}(\mathrm{n}, \mathrm{f})^{243} \mathrm{Am}$ and ${ }^{242 \mathrm{~m}} \mathrm{Am}(\mathrm{n}, \mathrm{f}){ }^{243} \mathrm{Am}$. These cross sections were obtained in a pure Maxwellian neutron flux. They complement previous measurements done on ${ }^{241} \mathrm{Am}$ [14] and ${ }^{243} \mathrm{Am}$ [6] capture cross sections. The obtained effective and $25.3 \mathrm{meV}$ cross sections are shown in table 4 .

The most striking discrepancy between our results and recent evaluated data libraries concerns the ${ }^{242} \mathrm{Cm}(\mathrm{n}, \gamma){ }^{243} \mathrm{Cm}$ cross section which is underestimated by about $12 \%$ in the libraries. For other reactions, discrepancies are below $10 \%$.

Table 4. Effective and $25.3 \mathrm{meV}$ measured cross sections.

\begin{tabular}{lll}
\hline Reactions & $\langle\sigma\rangle(\mathrm{b})$ & $\sigma_{0}(\mathrm{~b})$ \\
\hline${ }^{241} \mathrm{Am}(\mathrm{n}, \gamma)^{242} \mathrm{Am}$ & $609 \pm 20$ & $705 \pm 23$ \\
Branching ratio & & $0.8947 \pm 0.0038$ \\
${ }^{242 \mathrm{~m}} \mathrm{Am}(\mathrm{n}, \gamma)^{243} \mathrm{Am}$ & $1131 \pm 108$ & $1161 \pm 111$ \\
${ }^{242 \mathrm{gs}} \mathrm{Am}(\mathrm{n}, \mathrm{f})$ & $2223 \pm 215$ & $2644.07 \pm 257$ \\
${ }^{242 \mathrm{~m}} \mathrm{Am}(\mathrm{n}, \mathrm{f})$ & $6526 \pm 625$ & $6855.97 \pm 657$ \\
${ }^{242} \mathrm{Cm}(\mathrm{n}, \gamma)^{243} \mathrm{Cm}$ & $19.4 \pm 1.5$ & $19.1 \pm 1.5$ \\
\hline
\end{tabular}

\subsection{Curium transmutation chain}

${ }^{244} \mathrm{Cm}(\mathrm{n}, \gamma)$ and ${ }^{245} \mathrm{Cm}(\mathrm{n}, \mathrm{f})$ were measured very recently by means of TDFC in the V4 channel. A $(39.46 \pm 0.4) \mu \mathrm{g}$ mass of curium containing $66.9 \%$ of ${ }^{244} \mathrm{Cm}, 3.8 \%$ of ${ }^{245} \mathrm{Cm}$ and 


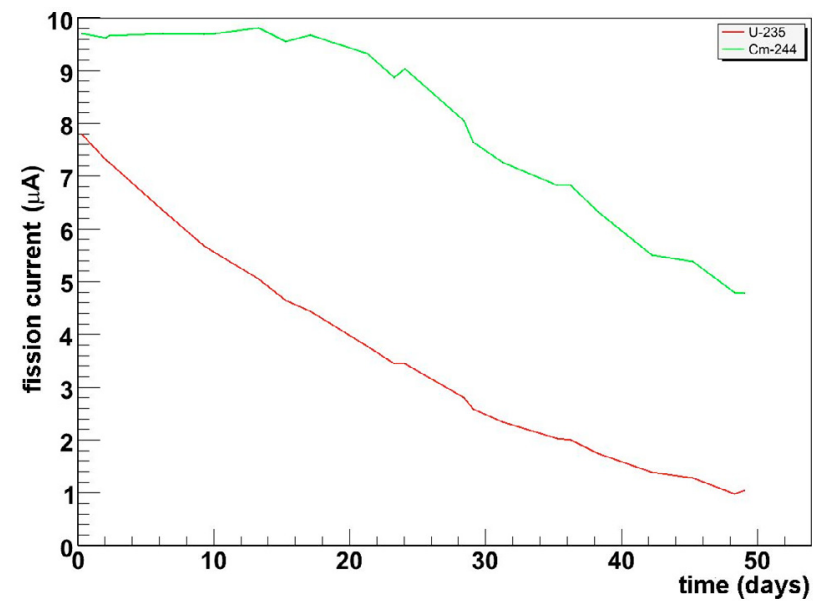

Fig. 3. Fission currents measured in the ${ }^{244} \mathrm{Cm}$ and ${ }^{235} \mathrm{U}$ fission chambers.

$23.6 \%$ of ${ }^{240} \mathrm{Pu}$ was irradiated. The isotopic concentration was determined before irradiation by mass spectrometry with a precision better than $1 \%$.

Figure 3 shows the evolution of fission currents for both curium and ${ }^{235} \mathrm{U}$ chambers. Currents were recorded every two seconds in order to determine the neutron flux evolution with good precision. Measured currents were then fitted with the MERCS code taking into account the evolution of ${ }^{240} \mathrm{Pu}$ and propagating the associated errors. ${ }^{240} \mathrm{Pu}$ isotope is the main responsible for the bump in the curium fission current between 10 and 30 days. Preliminary results are given in table 5.

Table 5. Preliminary effective and $25.3 \mathrm{meV}$ measured cross sections.

\begin{tabular}{lll}
\hline Reactions & $\langle\sigma\rangle(\mathrm{b})$ & $\sigma_{0}(\mathrm{~b})$ \\
\hline${ }^{244} \mathrm{Cm}(\mathrm{n}, \gamma)^{245} \mathrm{Cm}$ & $28.7 \pm 3.4$ & $20.8 \pm 2.5$ \\
${ }^{245} \mathrm{Cm}(\mathrm{n}, \mathrm{f})$ & $1400 \pm 29$ & $2012 \pm 61$ \\
\hline
\end{tabular}

\section{Conclusions}

The Mini-INCA set-up installed at the ILL High Flux Reactor is proven to be well suited for transmutation studies in high thermal neutron fluxes. The two dedicated apparatus are complementary and allow microscopic as well as macroscopic measurements even of rare short-lived isotopes. The capture and fission cross sections we have measured generally show good agreements with the most recent measurements. However, large discrepancies with evaluated data libraries have been observed in a few cases. The new TDFC developed for high neutrons fluxes are a very promising tool for on-line transmutation and incineration studies. They make possible to check on-line the evolution of an actinide in a given flux and also to monitor the variations of the neutron flux. Moreover, benefiting from the fission chamber technology, flux and incineration measurements have also be done at the MEGAPIE spallation target.

Authors are grateful to ILL technicians for their great help in the preparation of experiments. This work was partially supported by the GDR GEDEPEON (France).

\section{References}

1. G. Aliberti et al., Ann. Nucl. Energy 33, 700 (2006).

2. O. Bringer et al., Ann. Nucl. Energy (to be submitted).

3. G. Fioni et al., J. Nucl. Sci. Technol. Suppl. 2, issue 2, 876 (2002).

4. A. Letourneau et al., Ann. Nucl. Energy 33, 377 (2006).

5. S. Panebianco et al. (these proceedings).

6. F. Marie et al., Nucl. Instrum. Meth. A 556, 547 (2006).

7. M. Fadil et al., Ph.D. thesis, INP Grenoble, 2003; Nucl. Instrum. Meth. A (2002).

8. S. Chabod, Ph.D. thesis, University of Paris XI, 2006; S. Chabod et al., Nucl. Instrum. Meth. A 566, 633 (2006).

9. see http://www-dapnia.cea.fr/Sphn/MNM/Modelisation/

10. D. Ridikas et al., Proceedings of the $6^{\text {th }}$ OECD/NEA Information Exchange Meeting on Actinide and Fission Partitioning and Transmutation, 11-13 December 2000, Madrid, Spain.

11. A. Letourneau et al., CEA/DSM/SPhN-03-243 report, 2003 (unpublished).

12. O. Bringer et al., Nucl. Instrum. Meth. (to be submitted).

13. A. Letourneau et al., Nucl. Phys. A (to be submitted).

14. G. Fioni et al., Nucl. Phys. A 693, 546 (2001). 